\title{
Semigroups generated by elliptic operators in non-divergence form on $C_{0}(\Omega)$
}

\author{
Wolfgang AREndt AND Reiner Michael ScHÄtZle
} Abstract. Let $\Omega \subset \mathbb{R}^{n}$ be a bounded open set satisfying the uniform exterior cone
condition. Let $\mathcal{A}$ be a uniformly elliptic operator given by

$$
\mathcal{A} u=\sum_{i, j=1}^{n} a_{i j} \partial_{i j} u+\sum_{j=1}^{n} b_{j} \partial_{j} u+c u
$$

where

$$
a_{j i}=a_{i j} \in C(\bar{\Omega}) \text { and } b_{j}, c \in L^{\infty}(\Omega), c \leq 0 .
$$

We show that the realization $A_{0}$ of $\mathcal{A}$ in

$$
C_{0}(\Omega):=\left\{u \in C(\bar{\Omega}): u_{\left.\right|_{\partial \Omega}}=0\right\}
$$

given by

$$
\begin{aligned}
D\left(A_{0}\right) & :=\left\{u \in C_{0}(\Omega) \cap W_{\mathrm{loc}}^{2, n}(\Omega): \mathcal{A} u \in C_{0}(\Omega)\right\} \\
A_{0} u & :=\mathcal{A} u
\end{aligned}
$$

generates a bounded holomorphic $C_{0}$-semigroup on $C_{0}(\Omega)$. The result is in particular true if $\Omega$ is a Lipschitz domain. So far the best known result seems to be the case where $\Omega$ has $C^{2}$-boundary [12, Section 3.1.5]. We also study the elliptic problem

$$
\begin{aligned}
& -\mathcal{A} u=f \\
& u_{\left.\right|_{\partial \Omega}}=g .
\end{aligned}
$$

Mathematics Subject Classification (2010): 35K20 (primary); 35J25, 47D06 (secondary).

\section{Introduction}

The aim of this paper is to study elliptic and parabolic problems for operators in non-divergence form with continuous second order coefficients and to prove existence (and uniqueness) of solutions which are continuous up to the boundary of the 
domain. Such parabolic equations are a classical subject in pde. The consideration of operators in non-divergence form occurs naturally in many situations, for example in the context of stochastic differential equations, where the corresponding parabolic equation is frequently called Kolmogorov's equation [8, page 4], or in the non-linear theory, where regularity results play an important role. Here we are interested in continuity up to the boundary. More precisely, we want to investigate when an elliptic operator generates an analytic semigroup on

$$
C_{0}(\Omega):=\left\{u \in C(\bar{\Omega}): u_{\left.\right|_{\partial \Omega}}=0\right\}
$$

where $\Omega \subset \mathbb{R}^{n}$ is a bounded, open set. We refer to Lunardi's monograph [12] for a systematic theory of holomorphic semigroups generated by elliptic operators. Also in this monograph such semigroups are studied on spaces of continuous functions.

Throughout this paper $\Omega$ is a bounded open set in $\mathbb{R}^{n}, n \geq 2$, with boundary $\partial \Omega$. We consider the operator $\mathcal{A}$ given by

$$
\mathcal{A} u:=\sum_{i, j=1}^{n} a_{i j} \partial_{i j} u+\sum_{j=1}^{n} b_{j} \partial_{j} u+c u
$$

with real-valued coefficients $a_{i j}, b_{j}, c$ satisfying

$$
\begin{aligned}
& b_{j} \in L^{\infty}(\Omega), j=1, \ldots, n, c \in L^{\infty}(\Omega), c \leq 0 \\
& a_{i j} \in C(\Omega) \cap L^{\infty}(\Omega), a_{i j}=a_{j i}, \\
& \sum_{i, j=1}^{n} a_{i j}(x) \xi_{i} \xi_{j} \geq \Lambda|\xi|^{2} \quad\left(x \in \Omega, \xi \in \mathbb{R}^{n}\right)
\end{aligned}
$$

where $\Lambda>0$ is a fixed constant.

Only for part of the results we need that the $a_{i j}$ are continuous up to the boundary (as is assumed in the Abstract). Of course, the assumption that $a_{i j}=a_{j i}$ is no restriction of generality (replacing $a_{i j}$ by $\frac{a_{i j}+a_{j i}}{2}$ otherwise).

Our best results are obtained under the hypothesis that $\Omega$ satisfies the uniform exterior cone condition (and thus in particular if $\Omega$ has Lipschitz boundary). Then we show that for each $f \in L^{n}(\Omega), g \in C(\partial \Omega)$ there exists a unique $u \in C(\bar{\Omega}) \cap$ $W_{\text {loc }}^{2, n}(\Omega)$ such that

$$
\text { (E) }\left\{\begin{array}{l}
-\mathcal{A} u=f \\
u_{\left.\right|_{\partial \Omega}}=g .
\end{array}\right.
$$

(Corollary 3.4). This result is proved with the help of Alexandrov's maximum principle (which is responsible for the choice of $p=n$ ) and other standard results for elliptic second order differential operators (put together in the appendix). Our main concern is the parabolic problem

$$
(P)\left\{\begin{aligned}
u_{t} & =\mathcal{A} u \\
u(0, \cdot) & =u_{0} \\
u(t, x) & =0 \quad x \in \partial \Omega, t>0 .
\end{aligned}\right.
$$


with Dirichlet boundary conditions. Under the uniform exterior cone condition, we show that the realization $A_{0}$ of $\mathcal{A}$ in $C_{0}(\Omega)$ given by

$$
\begin{aligned}
D\left(A_{0}\right) & :=\left\{v \in C_{0}(\Omega) \cap W_{\mathrm{loc}}^{2, n}(\Omega): \mathcal{A} v \in C_{0}(\Omega)\right\} \\
A_{0} v & :=\mathcal{A} v
\end{aligned}
$$

generates a bounded, $C_{0}$-semigroup on $C_{0}(\Omega)$, which is holomorphic, if in addition $a_{i j} \in C(\bar{\Omega})$. This improves the known results, which are presented in the monographe of Lunardi [12, Corollary 3.1.21] for $\Omega$ of class $C^{2}$ (and $b_{j}, c$ uniformly continuous).

We would like to mention one particular reason why continuity at the boundary is important. If one considers semilinear equations the semilinear term $f(u)$ is usually treated by a fixed point argument. For this it is useful that the composition mapping $u \mapsto f(u)$ is locally Lipschitz continuous on the underlying function space $X$, where $f: \mathbb{R} \rightarrow \mathbb{R}$ defines the semilinear term. If $X=L^{p}$, then this is merely the case if $f$ is already globally Lipschitz continuous, a condition which is too strong for many applications. However, on $C_{0}(\Omega)$ local Lipschitz continuity of $f$ implies the same property of the composition operator. We refer to the monograph [7] of Cazenave-Haraux where the space $C_{0}(\Omega)$ is used for this purpose.

For applications, the improvement from $C^{2}$ to Lipschitz boundary is certainly the most important case of our results. But it is natural to ask for the minimal hypotheses on coefficients and boundary. It is interesting that one may relax the condition at the boundary if more regularity of the coefficients is assumed and vice versa. Three regularity properties at the boundary are considered in this paper: Lipschitz boundary (as the strongest concerning our results), the uniform exterior cone condition and Wiener regularity as the weakest property. Concerning the coefficients $a_{i j}$ we assume global Lipschitz-continuity as the strongest, Dini continuity, Hölder continuity, continuity up to the boundary, and just continuity in the interior as the weakest possible assumption, formulated as our standing hypothesis above. The interplay among these properties seems interesting to us and we devote some particular attention to it. For example, our weakest condition at the boundary, namely Wiener regularity, suffices for the generation of a holomorphic semigroup on $C_{0}(\Omega)$ and for well-posedness of $(E)$ if the second order coefficients are globally Lipschitz continuous. On the other hand if merely $a_{i j} \in C(\bar{\Omega})$ then we need that $\Omega$ fulfills the exterior cone condition.

In order to show the existence of a semigroup, we have to study the elliptic problem $(E)$ before (to verify the range condition in the Hille-Yosida theorem). At first we consider the Poisson equation with homogeneous boundary data $g=0$ (Section 1). This is done merely on the basis of the standard elliptic theory, as presented in the monograph of Gilbarg-Trudinger [9], and leads in relatively elementary way to two important cases $\left(a_{i j}\right.$ Lipschitz, $\Omega$ Wiener-regular in Theorem 1.1 and uniform exterior cone condition for $\Omega$ and $a_{i j}$ merely bounded and continuous in the interior in Theorem 1.4).

In Section 2 we show that the Poisson problem is equivalent to the Dirichlet problem (i.e., $f=0$ ). This is of independent interest. But it also allows us to 
use results of Krylov [10] to obtain a further case for the Generation Theorem 3.1 (namely $a_{i j}$ Dini-continuous and $\Omega$ merely Wiener regular). The main results on the parabolic equations are obtained in Section 3.

We conclude the introduction by mentioning which results are known if an elliptic operator in divergence form with measurable bounded coefficients is considered (instead of our operator in non-divergence form). Such operator defines a mapping $\mathcal{A}: H_{\mathrm{loc}}^{1}(\Omega) \rightarrow \mathcal{D}(\Omega)^{\prime}$. If $\Omega$ is Wiener regular, then for each $f \in$ $L^{p}(\Omega), p>\frac{n}{2}$, and each $g \in C(\partial \Omega)$ there exists a unique $u \in C(\bar{\Omega}) \cap H_{\text {loc }}^{1}(\Omega)$ such that $\mathcal{A} u=f, u_{\left.\right|_{\partial \Omega}}=g$ (see [9, Theorem 8.31]). However, in general the solution is no longer in $W_{\text {loc }}^{2, n}(\Omega)$ as in the case considered in the present paper. Concerning the generation theorem, one knows that the part $A_{0}$ of $\mathcal{A}$ in $C_{0}(\Omega)$, defined on the domain $D\left(A_{0}\right):=\left\{u \in H_{\mathrm{loc}}^{1}(\Omega) \cap C_{0}(\Omega): \mathcal{A} u \in C_{0}(\Omega)\right\}$ generates holomorphic $C_{0^{-}}$ semigroup on $C_{0}(\Omega)$, see [1], again on the sole hypothesis that $\Omega$ is Wiener regular. The same remains true for operators in non-divergence form of the type $m \cdot \Delta$; i.e. in the very special case of isotropic coefficients $\left(a_{i j}(x)=m(x), i, j=1, \ldots, n\right)$. This remains even true in the case where $m(x)$ degenerates at the boundary (see [3]).

\section{The Poisson problem}

We consider the bounded open set $\Omega \subset \mathbb{R}^{n}$ and the elliptic operator $\mathcal{A}$ from the introduction. At first we consider the case where the second order coefficients are Lipschitz continuous. Then we merely need a very mild regularity condition on $\Omega$. We say that $\Omega$ is Wiener regular if for each $g \in C(\partial \Omega)$ there exists a solution $u \in C^{2}(\Omega) \cap C(\bar{\Omega})$ of the Dirichlet problem

$$
\begin{aligned}
\Delta u & =0 \\
u_{\mid \partial \Omega} & =g .
\end{aligned}
$$

The name comes from Wiener's characterization via capacity $[9,(2.37)]$. If $\Omega$ satisfies the exterior cone condition, then $\Omega$ is Wiener regular.

Theorem 2.1. Assume that the second order coefficients $a_{i j}$ are globally Lipschitz continuous. If $\Omega$ is Wiener regular, then for each $f \in L^{n}(\Omega)$, there exists a unique $u \in W_{\mathrm{loc}}^{2, n}(\Omega) \cap C_{0}(\Omega)$ such that

$$
-\mathcal{A} u=f .
$$

The point is that for Lipschitz continuous $a_{i j}$ the operator $\mathcal{A}$ may be written in divergence form. This is due to the following lemma.

Lemma 2.2. Let $h: \Omega \rightarrow \mathbb{R}$ be Lipschitz continuous. Then $h \in W^{1, \infty}(\Omega)$. In particular, $h u \in W^{1,2}(\Omega)$ for all $u \in W^{1,2}(\Omega)$ and $\partial_{j}(h u)=\left(\partial_{j} h\right) u+h \partial_{j} u$.

Proof. One can extend $h$ to a Lipschitz function on $\mathbb{R}^{n}$ (without increasing the Lipschitz constant, see [15]). Now the result follows from [8, 5.8 Theorem 4]. 
Proof of Theorem 2.1. We assume that $\Omega$ is Dirichlet regular. Uniqueness follows from Aleksandrov's maximum principle Theorem A.1. In order to solve the problem we replace $\mathcal{A}$ by an operator in divergence form in the following way. Let $\tilde{b}_{j}:=b_{j}-\sum_{i=1}^{n} \partial_{i} a_{i j}, j=1, \ldots, n$. Then $\tilde{b}_{j} \in L^{\infty}(\Omega)$. Consider the elliptic operator $\mathcal{A}_{d}$ in divergence form given by

$$
\mathcal{A}_{d} u=\sum_{i, j=1}^{n} \partial_{i}\left(a_{i j} \partial_{j} u\right)+\sum_{j=1}^{n} \tilde{b}_{j} \partial_{j} u+c u .
$$

Let $f \in L^{n}(\Omega)$. By [9, Theorem 8.31] or [1, Corollary 4.6] there exists a unique $u \in C_{0}(\Omega) \cap W_{\mathrm{loc}}^{1,2}(\Omega)$ such that $-\mathcal{A}_{d} u=f$ weakly, i.e.,

$$
\sum_{i, j=1}^{d} \int_{\Omega} a_{i j} \partial_{j} u \partial_{i} v-\sum_{j=1}^{d} \int_{\Omega} \tilde{b}_{j} \partial_{j} u v-\int_{\Omega} c u v=\int_{\Omega} f v
$$

for all $v \in \mathcal{D}(\Omega)$ (the space of all test functions). We mention in passing that $u \in$ $W_{0}^{1,2}(\Omega)$ by [1, Lemma 4.2]. For our purposes, it is important that $u \in W_{\text {loc }}^{2,2}(\Omega)$ by Friedrich's theorem [9, Theorem 8.8]. Here we use again that the $a_{i j}$ are uniformly Lipschitz continuous but do not need any further hypothesis on $b_{j}$ and $c$. It follows from Lemma 2.2 that $a_{i j} \partial_{j} u \in W_{\mathrm{loc}}^{1,2}(\Omega)$ and $\partial_{i}\left(a_{i j} \partial_{j} u\right)=\left(\partial_{i} a_{i j}\right) \partial_{j} u+a_{i j} \partial_{i j} u$. Thus $\mathcal{A}_{d} u=\mathcal{A} u$. Now it follows from [9, Lemma 9.16] that $u \in W_{\text {loc }}^{2, n}(\Omega)$.

Remark 2.3. Theorem 1.1 is formulated as it is needed for the main result in Section 4. Actually, more is true. Let $f \in L^{p}(\Omega)$ where $p>\frac{n}{2}$. Then there exists a unique $u \in W_{\mathrm{loc}}^{2, p}(\Omega) \cap C_{0}(\Omega)$ such that $-\mathcal{A} u=f$. Uniqueness follows from [9, Lemma 9.16]: If $\mathcal{A} u=0$ then $u \in W_{\text {loc }}^{2, q}(\Omega)$ for all $q \geq p$. So we can apply Alexandrov's maximum principle. In order to prove existence, we choose $f_{k} \in L^{n}(\Omega)$ converging to $f$ in $L^{p}(\Omega)$ as $k \rightarrow \infty$. By the proof of Theorem 2.1 we find $u_{k} \in C_{0}(\Omega) \cap W_{\text {loc }}^{2, n}(\Omega)$ such that $-\mathcal{A}_{d} u_{k}=-\mathcal{A} u_{k}=f_{k}$. Choose $\Omega_{m} \subset \subset \Omega$ such that $\overline{\Omega_{m}} \subset \Omega_{m+1}, \bigcup_{m \in \mathbb{N}} \Omega_{m}=\Omega$. Then clearly $u_{k} \in W^{2,2}\left(\Omega_{m}\right) \subset W^{1,2}\left(\Omega_{m}\right)$. Thus [9, Theorem 8.16] shows that

$$
\left\|u_{k}-u_{\ell}\right\|_{L^{\infty}\left(\Omega_{m}\right)} \leq\left\|u_{k}-u_{\ell}\right\|_{C\left(\partial \Omega_{m}\right)}+C\left\|f_{k}-f_{\ell}\right\|_{L^{p}\left(\Omega_{m}\right)}
$$

for all $m \in \mathbb{N}$. Letting $m \rightarrow \infty$ it follows that

$$
\left\|u_{k}-u_{\ell}\right\|_{L^{\infty}(\Omega)} \leq C\left\|f_{k}-f_{\ell}\right\|_{L^{p}(\Omega)} .
$$

Thus $u:=\lim _{k \rightarrow \infty} u_{k}$ converges in $C_{0}(\Omega)$. By the Calderon-Zygmund estimate Theorem A.2

$$
\left\|u_{k}-u_{\ell}\right\|_{W^{2, p}\left(B_{\varrho}\right)} \leq c_{\varrho}(p)\left(\left\|u_{k}-u_{\ell}\right\|_{L^{p}\left(B_{2 \varrho}\right)}+\left\|f_{k}-f_{\ell}\right\|_{L^{p}\left(B_{2 \varrho}\right)}\right)
$$

whenever $\bar{B}_{2 \varrho} \subset \Omega$. Thus $u \in W_{\mathrm{loc}}^{2, p}(\Omega)$ and $\lim _{k \rightarrow \infty} u_{k}=u$ in $W_{\text {loc }}^{2, p}(\Omega)$. It follows that $-\mathcal{A} u=\lim _{k \rightarrow \infty}-\mathcal{A} u_{k}=f$. 
Now we return to the general assumption $a_{i j} \in C(\Omega) \cap L^{\infty}(\Omega)$ and do no longer assume that the $a_{i j}$ are Lipschitz continuous. The following result is stated in [9, Theorem 9.30] with a sketchy proof referring to the Perron method. We include a direct proof based on our Theorem 2.1, for the convenience of the reader.

Theorem 2.4. Assume that $\Omega$ satisfies the uniform exterior cone condition. Then for all $f \in L^{n}(\Omega)$ there exists a unique $u \in C_{0}(\Omega) \cap W_{\text {loc }}^{2, n}(\Omega)$ such that $-\mathcal{A} u=f$.

Proof. As for Theorem 2.1 we merely have to prove existence of a solution. We extend $a_{i j}$ by $\delta_{i j}$ outside $\Omega$ and mollify to obtain $a_{i j}^{k} \in C^{\infty}\left(\mathbb{R}^{n}\right) \subset \operatorname{Lip}(\Omega)$ uniformly elliptic and bounded, converging to $a_{i j}$ locally uniformly in $\Omega$. Let $\mathcal{A}_{k}$ be the elliptic operator with the second order coefficients $a_{i j}$ of $\mathcal{A}$ replaced by $a_{i j}^{k}$. Let $f \in L^{n}(\Omega)$. By Theorem 2.1, for each $k \in \mathbb{N}$ there exists a unique $u_{k} \in W_{\text {loc }}^{2, n}(\Omega) \cap C_{0}(\Omega)$ such that $-\mathcal{A}_{k} u_{k}=f$. By Hölder regularity (Theorem A.3) there exists a constant $c$ which does not depend on $k \in \mathbb{N}$ such that

$$
\left\|u_{k}\right\|_{C^{\alpha}(\Omega)} \leq c\left(\|f\|_{L^{n}(\Omega)}+\left\|u_{k}\right\|_{L^{n}(\Omega)}\right) .
$$

By Aleksandrov's maximum principle $\left\|u_{k}\right\|_{L^{\infty}(\Omega)} \leq c_{1}\|f\|_{L^{n}(\Omega)}$ for all $k \in \mathbb{N}$ and some constant $c_{1}$. Thus $\left(u_{k}\right)_{k \in \mathbb{N}}$ is bounded in $C^{\alpha}(\Omega)$. By the Arzela-Ascoli theorem we may assume that $u_{k}$ converges uniformly to $u \in C_{0}(\Omega)$ as $k \rightarrow \infty$ (passing to a subsequence if necessary). Let $\overline{B_{2 \varrho}} \subset \Omega$ where $B_{2 \varrho}$ is a ball of radius $2 \varrho$. Since the modulus of continuity of the $a_{i j}^{k}$ is locally bounded in $\Omega$, by the interior Calderon-Zygmund estimate Theorem A.2

$$
\left\|u_{k}\right\|_{W^{2, n}\left(B_{\varrho}\right)} \leq c_{2}\left(\left\|u_{k}\right\|_{L^{n}\left(B_{2 \varrho}\right)}+\|f\|_{L^{n}\left(B_{2 \varrho}\right)}\right)
$$

for all $k \in \mathbb{N}$ and some constant $c_{2}$, which depends on $\varrho$ and the position of $B_{\varrho}$. It follows from reflexivity that $u \in W^{2, n}\left(B_{\varrho}\right)$ and $u_{k} \rightarrow u$ in $W^{2, n}\left(B_{\varrho}\right)$ as $k \rightarrow \infty$ after extraction of a subsequence. Since $-\mathcal{A}_{k} u_{k}=f$, it follows that $-\mathcal{A} u=f$. In fact, since $u_{k} \rightarrow u$ weakly in $W^{2, n}\left(B_{\varrho}\right)$, it follows that $\partial_{i j} u_{k} \rightarrow \partial_{i j} u$ in $L^{n}\left(B_{\varrho}\right)$ as $k \rightarrow \infty$. Thus sup $\left\|\partial_{i j} u_{k}\right\|_{L^{n}\left(B_{\varrho}\right)}<\infty$. It follows that

$$
\left(a_{i j}^{k}-a_{i j}\right) \partial_{i j} u_{k} \rightarrow 0 \text { in } L^{n}\left(B_{\varrho}\right) \text { as } k \rightarrow \infty
$$

and consequently $a_{i j}^{k} \partial_{i j} u_{k} \rightarrow a_{i j} \partial_{i j} u$ in $L^{n}\left(B_{\varrho}\right)$.

\section{The Dirichlet problem}

In this section we show the equivalence between well-posedness of the Poisson problem

$$
\begin{aligned}
& -\mathcal{A} u=f \\
& u_{\left.\right|_{\partial \Omega}}=0
\end{aligned}
$$


and the Dirichlet problem

$$
\begin{aligned}
& \mathcal{A} u=0 \\
& u_{\left.\right|_{\partial \Omega}}=g
\end{aligned}
$$

where $f \in L^{n}(\Omega)$ and $g \in C(\partial \Omega)$ are given. We consider the operator $\mathcal{A}$ defined in the previous section and define its realization $A$ in $L^{n}(\Omega)$ (recall that $\Omega \subset \mathbb{R}^{n}$ ) by

$$
\begin{aligned}
D(A) & :=\left\{u \in C_{0}(\Omega) \cap W_{\mathrm{loc}}^{2, n}(\Omega): \mathcal{A} u \in L^{n}(\Omega)\right\} \\
A u & :=\mathcal{A} u
\end{aligned}
$$

Thus the Poisson problem can be formulated in a more precise way by asking under which conditions $A$ is invertible (i.e. bijective from $D(A)$ to $L^{n}(\Omega)$ with bounded inverse $\left.A^{-1}: L^{n}(\Omega) \rightarrow L^{n}(\Omega)\right)$. By Aleksandrov's maximum principle, $A$ is always injective. Note that for $\mu>0$, the operator $A-\mu:=A-\mu I$ has the same form as $A$ (the order-0-coefficient $c$ being just replaced by $c-\mu$ ).

Proposition 3.1. The operator $A$ is closed, injective and has closed range. If $A$ is invertible, then the operator $\tilde{A}$ obtained by replacing the coefficient of order 0 by another coefficient $0 \geq \tilde{c} \in L^{\infty}(\Omega)$ is also invertible. In particular, if $A-\mu_{0}$ has dense range in $L^{n}(\Omega)$ for some $\mu_{0} \geq 0$, then $A-\mu$ is invertible for all $\mu \geq 0$,

Proof. By the Aleksandrov maximum principle (Theorem A.1) there exists a constant $c_{1}>0$ such that

$$
\|u\|_{\infty} \leq c_{1}\|\mu u-A u\|_{L^{n}(\Omega)}
$$

for all $u \in D(A), \mu \geq 0$. In order to show that $A$ is closed, let $u_{k} \in D(A)$ such that $u_{k} \rightarrow u$ in $L^{n}(\Omega)$ and $A u_{k} \rightarrow f$ in $L^{n}(\Omega)$. It follows from (3.1) that $u \in C_{0}(\Omega)$ and $\lim _{k \rightarrow \infty} u_{k}=u$ in $C_{0}(\Omega)$. Let $B_{2 \varrho}$ be a ball of radius $2 \varrho$ such that $\overline{B_{2 \varrho}} \subset \Omega$. By the Calderon-Zygmund estimate (Theorem A.2)

$$
\left\|u_{k}-u_{\ell}\right\|_{W^{2, n}\left(B_{\varrho}\right)} \leq c_{\varrho}\left(\left\|u_{k}-u_{\ell}\right\|_{L^{n}\left(B_{2 \varrho}\right)}+\left\|A\left(u_{k}-u_{\ell}\right)\right\|_{L^{n}\left(B_{2 \varrho}\right)}\right) .
$$

It follows that $\left(u_{k}\right)_{k \in \mathbb{N}}$ converges to $u$ in $W^{2, n}\left(B_{\varrho}\right)$. Consequently $\mathcal{A} u_{k} \rightarrow \mathcal{A} u$ in $L^{n}\left(B_{\varrho}\right)$. Thus $\mathcal{A} u=f$ on $B_{\varrho}$. Since the ball is arbitrary, it follows that $u \in D(A)$ and $A u=f$. We have thus shown that $A$ is closed. Now it follows from (3.1) that the range of $A$ is closed. Next we show that invertibility is independent of the choice of the coefficient of order 0 . Assume that $A$ is invertible. Consider $A(t)=A+t(\tilde{c}-c)$, which corresponds to replacing $c$ by $c+t(\tilde{c}-c)$, which is still $\leq 0$ for $t \in[0,1]$. Moreover, $D(A(t))=D(A)$. We want to show that $A(1)$ is invertible. The set

$$
M:=\{t \in[0,1]: A(t) \text { is invertible }\}
$$


contains $t=0$ by hypothesis. Since $u \mapsto(\tilde{c}-c) u$ is a bounded operator on $L^{n}(\Omega)$, the set $M$ is open. In fact, assume that $A\left(t_{0}\right)$ is invertible. If

$$
\left|t-t_{0}\right|\|c-\tilde{c}\|_{L^{\infty}(\Omega)}\left\|A\left(t_{0}\right)^{-1}\right\|_{\mathcal{L}\left(L^{n}(\Omega)\right)}<1,
$$

then $S:=I-\left(t-t_{0}\right)(c-\tilde{c}) A\left(t_{0}\right)^{-1}$ is invertible in $\mathcal{L}\left(L^{n}(\Omega)\right)$ by the Neumann series. It follows that $A(t)=S A\left(t_{0}\right)$ is invertible.

It remains to show that $M$ is closed. Let $t_{k} \in M$ such that $\lim _{k \rightarrow \infty} t_{k}=t_{0}$. Let $f \in L^{n}(\Omega)$. There exist $u_{k} \in D(A)$ such that $A\left(t_{k}\right) u_{k}=f$. By Aleksandrov's maximum principle we have

$$
\left\|u_{k}\right\|_{\infty} \leq c_{1}\|f\|_{L^{n}(\Omega)} \quad(k \in \mathbb{N}) .
$$

Moreover,

$$
\begin{aligned}
A\left(t_{k}\right)\left(u_{k}-u_{\ell}\right) & =\left(A\left(t_{\ell}\right)-A\left(\left(t_{k}\right)\right) u_{\ell}\right. \\
& =\left(t_{\ell}-t_{k}\right)(\tilde{c}-c) u_{\ell} .
\end{aligned}
$$

From Aleksandrov's maximum principle we conclude now that

$$
\left\|u_{k}-u_{\ell}\right\|_{\infty} \leq c_{1}^{2}\left|t_{\ell}-t_{k}\right|\|\tilde{c}-c\|_{\infty}\|f\|_{L^{n}(\Omega)} .
$$

It follows that $\lim _{k \rightarrow \infty} u_{k}=u$ exists in $C_{0}(\Omega)$. Observe that

$$
\begin{aligned}
A u_{k} & =A\left(t_{k}\right) u_{k}-t_{k}(\tilde{c}-c) u_{k} \\
& =f-t_{k}(\tilde{c}-c) u_{k}
\end{aligned}
$$

converges to $f-t_{0}(\tilde{c}-c) u$ in $C_{0}(\Omega)$ and hence in $L^{n}(\Omega)$. Since $A$ is closed, it follows that $u \in D(A)$ and $A u=f-t_{0}(\tilde{c}-c) u ; i . e ., A\left(t_{0}\right) u=f$. We have shown that $A\left(t_{0}\right)$ is surjective. As noted before, $A\left(t_{0}\right)$ is injective. Hence $t_{0} \in M$.

We call a function $u$ on $\Omega \mathcal{A}$-harmonic if $u \in W_{\text {loc }}^{2, p}(\Omega)$ for some $p>1$ and $\mathcal{A} u=0$. By [9, Theorem 9.16] each $\mathcal{A}$-harmonic function $u$ is in $\bigcap_{q>1} W_{\mathrm{loc}}^{2, q}(\Omega)$. Given $g \in C(\partial \Omega)$, the Dirichlet problem consists in finding an $\mathcal{A}$-harmonic function $u \in C(\bar{\Omega})$ such that $u_{\left.\right|_{\partial \Omega}}=g$. We say that $\Omega$ is $\mathcal{A}$-regular if for each $g \in C(\partial \Omega)$ there is a solution of the Dirichlet problem. Uniqueness follows from the maximum principle [9, Theorem 9.6]

$$
-\left\|u_{\left.\right|_{\partial \Omega}}^{-}\right\|_{C(\partial \Omega)} \leq u(x) \leq\left\|u_{\mid \partial \Omega}^{+}\right\|_{C(\partial \Omega)}
$$

for all $x \in \bar{\Omega}$, which holds for each $\mathcal{A}$-harmonic function $u \in C(\bar{\Omega})$. In particular,

$$
\|u\|_{C(\bar{\Omega})} \leq\|u\|_{C(\partial \Omega)}
$$


Theorem 3.2. If the operator $A$ is invertible then $\Omega$ is $\mathcal{A}$-regular. Conversely, if $a_{i j} \in C(\bar{\Omega})$ and $\Omega$ is $\mathcal{A}$-regular, then $A$ is invertible. lent.

Thus for $a_{i j} \in C(\bar{\Omega})$ well-posedness of the problems $(P)$ and $(D)$ are equiva-

For the proof we need the following lemma which we learnt from H. Amann. We are grateful to him for the permission to include it. Recall from the Introduction that we assume throughout that $a_{i j}=a_{j i}$.

Lemma 3.3. a) There exist $\tilde{a}_{i j} \in C^{b}\left(\mathbb{R}^{n}\right)$ such that $\tilde{a}_{i j}=\tilde{a}_{j i}, \tilde{a}_{i j}(x)=a_{i j}(x)$ if $x \in \Omega$ and

$$
\sum_{i, j=1}^{n} a_{i j}(x) \xi_{i} \xi_{j} \geq \frac{\Lambda}{2}|\xi|^{2}
$$

for all $\xi \in \mathbb{R}^{n}, x \in \Omega$.

b) There exist $a_{i j}^{k} \in C^{\infty}(\bar{\Omega})$ such that $a_{i j}^{k}=a_{j i}^{k}, \sum_{i, j=1}^{n} a_{i j}^{k}(x) \xi_{i} \xi_{j} \geq \frac{\Lambda}{2}|\xi|^{2}$ and $\lim _{k \rightarrow \infty} a_{i j}^{k}(x)=a_{i j}(x)$ uniformly on $\bar{\Omega}$.

Proof. a) Let $b_{i j}: \mathbb{R}^{n} \rightarrow \mathbb{R}$ be a bounded, continuous extension of $a_{i j}$ to $\mathbb{R}^{n}$. Replacing $b_{i j}$ by $\frac{b_{i j}+b_{j i}}{2}$, we may assume that $b_{i j}=b_{j i}$. Since the function $\varphi$ : $\mathbb{R}^{n} \times S^{1} \rightarrow \mathbb{R}$ given by $\varphi(x, \xi):=\sum_{i, j=1}^{n} b_{i j}(x) \xi_{i} \xi_{j}$ is continuous and $S^{1}:=\{\xi \in$ $\left.\mathbb{R}^{n}:|\xi|=1\right\}$ is compact, the set $\Omega_{1}:=\left\{x \in \mathbb{R}^{n}: \varphi(x, \xi)>\frac{\Lambda}{2}\right.$ for all $\left.\xi \in S^{1}\right\}$ is open and contains $\bar{\Omega}$. Let $0 \leq \varphi_{1}, \varphi_{2} \in C\left(\mathbb{R}^{n}\right)$ such that $\varphi_{1}(x)+\varphi_{2}(x)=1$ for all $x \in \mathbb{R}^{n}$ and $\varphi_{2}(x)=1$ for $x \in \mathbb{R}^{n} \backslash \Omega_{1}, \varphi_{1}(x)=1$ for $x \in \bar{\Omega}$. Then $\tilde{a}_{i j}:=\varphi_{1} b_{i j}+\frac{\Lambda}{2} \varphi_{2} \delta_{i j}$ fulfills the requirements.

b) Let $\left(\varrho_{k}\right)_{k \in \mathbb{N}}$ be a mollifier satisfying supp $\varrho_{k} \subset B_{1 / k}(0)$. Then $a_{i j}^{k}=\tilde{a}_{i j} *$ $\varrho_{k} \in C^{\infty}\left(\mathbb{R}^{n}\right)$ and $\lim _{k \rightarrow \infty} a_{i j}^{k}(x)=\tilde{a}_{i j}(x)=a_{i j}(x)$ uniformly in $x \in \bar{\Omega}$. If $\frac{1}{k}<$ $\operatorname{dist}\left(\partial \Omega_{1}, \Omega\right)$, then for $x \in \Omega, \xi \in \mathbb{R}^{n}$

$$
\begin{aligned}
\sum_{i, j=1}^{n} a_{i j}^{k}(x) \xi_{i} \xi_{j} & =\int_{|y|<1 / k} \sum_{i, j=1}^{n} \tilde{a}_{i j}(x-y) \xi_{i} \xi_{j} \varrho_{k}(y) d y \\
& \geq \frac{\Lambda}{2} \int_{|y|<1 / k} \varrho_{k}(y) d y=\frac{\Lambda}{2} .
\end{aligned}
$$

Proof of Theorem 3.2. a) Assume that $A$ is invertible.

First step. Let $g \in C(\partial \Omega)$ be of the form $g=G_{\mid \partial \Omega}$ where $G \in C^{2}(\bar{\Omega})$. Then $\mathcal{A} G \in L^{n}(\Omega)$. Let $v=A^{-1}(\mathcal{A} G)$, then $u:=G-v$ solves the Dirichlet problem for $g$. 
Second step. Let $g \in C(\partial \Omega)$ be arbitrary. Extending $g$ continuously and mollifying we find $g_{k} \in C(\partial \Omega)$ of the kind considered in the first step such that $g=\lim _{k \rightarrow \infty} g_{k}$ in $C(\partial \Omega)$. Let $u_{k} \in C(\bar{\Omega})$ be $\mathcal{A}$-harmonic satisfying $\left.u_{k}\right|_{\partial \Omega}=g_{k}$. By (3.3) $u:=$ $\lim _{k \rightarrow \infty} u_{k}$ exists in $C(\bar{\Omega})$. In particular, $\left.u\right|_{\partial \Omega}=g$. Let $\overline{B_{2 \varrho}} \subset \Omega$. Then by the Calderon-Zygmund estimate Theorem A.2

$$
\left\|u_{k}\right\|_{W^{2, p}\left(B_{\varrho}\right)} \leq c_{\varrho}\left\|u_{k}\right\|_{L^{p}\left(B_{2 \varrho}\right)} \leq c_{\varrho} c\left\|u_{k}\right\|_{C(\bar{\Omega})}
$$

(remember that $\mathcal{A} u_{k}=0$ ). Thus $\left(u_{k}\right)_{k \in \mathbb{N}}$ is bounded in $W^{2, p}\left(B_{\varrho}\right)$. Passing to a subsequence, we can assume that $u_{k} \rightarrow u$ in $W^{2, p}\left(B_{\varrho}\right)$. This implies that $\mathcal{A} u=0$ in $B_{\varrho}$. Since the ball is arbitrary, it follows that $u$ is $\mathcal{A}$-harmonic. Thus $u$ is a solution of the Dirichlet problem $(D)$.

b) Conversely, assume that $\Omega$ is $\mathcal{A}$-regular. Let $f \in L^{n}(\Omega)$. We want to find $u \in D(A)$ such that $A u=f$. Let $B$ be a ball containig $\bar{\Omega}$ and extend $f$ by 0 to $B$. Then by Theorem 2.4 we find $v \in C_{0}(B) \cap W_{\text {loc }}^{2, n}(B)$ such that $\tilde{\mathcal{A}} v=f$. Here $\tilde{\mathcal{A}}$ is an extension of $\mathcal{A}$ to the ball $B$ according to Lemma 3.3a). Let $g=v_{\left.\right|_{\partial \Omega}}$. Then by our assumption there exists an $\mathcal{A}$-harmonic function $w \in C(\bar{\Omega})$ such that $w_{\left.\right|_{\partial \Omega}}=g$. Let $u=v-w$. Then $u \in C_{0}(\Omega) \cap W_{\text {loc }}^{2, n}(\Omega)$ and $\mathcal{A} u=\mathcal{A} v=f$; i.e. $u \in D(A)$ and $A u=f$. We have shown that $A$ is surjective, which implies invertibility by Proposition 3.1.

Corollary 3.4. Assume that one of the following two conditions is satisfied:

a) $\Omega$ is Wiener regular and the coefficients $a_{i j}$ are globally Lipschitz continuous, or

b) the $a_{i j}$ are in $C(\bar{\Omega})$ and $\Omega$ satisfies the exterior cone condition.

Then $\Omega$ is $\mathcal{A}$-regular. More generally, for all $f \in L^{n}(\Omega), g \in C(\partial \Omega)$ there exists a unique $u \in C(\bar{\Omega}) \cap W_{\text {loc }}^{2, n}(\Omega)$ satisfying

$$
\begin{aligned}
-\mathcal{A} u & =f \\
u_{\left.\right|_{\partial \Omega}} & =g .
\end{aligned}
$$

Proof. Since $A$ is closed by Proposition 3.1 it follows from Theorem 2.1 (in the case a)) and from Theorem 2.4 (in the case b)) that $A$ is invertible. Thus $\Omega$ is $\mathcal{A}$ regular by Theorem 3.2. Let $f \in L^{n}(\Omega), g \in C(\partial \Omega)$. Since $\Omega$ is $\mathcal{A}$-regular, there exists an $\mathcal{A}$-harmonic function $u_{1} \in C(\bar{\Omega})$ such that $u_{1_{\left.\right|_{\partial} \Omega}}=g$. Since $A$ is invertible, there exists a function $u_{0} \in C_{0}(\Omega) \cap W_{\text {loc }}^{2, n}(\Omega)$ such that $-\mathcal{A} u_{0}=f$. Let $u:=u_{0}+u_{1}$. Then $u \in C(\bar{\Omega}) \cap W_{\text {loc }}^{2, n}(\Omega), u_{\left.\right|_{\partial \Omega}}=g$ and $-\mathcal{A} u=f$. Uniqueness follows from Theorem A.1.

For the Laplacian $\mathcal{A}=\Delta, \Delta$-regularity is Wiener regularity as defined in the beginning of Section 1 . It is a most interesting question how $\mathcal{A}$-regularity and $\Delta$ regularity are related. In general it is not true that $\mathcal{A}$-regularity implies Wiener 
regularity. In fact, K. Miller [14] gives an example of an elliptic operator $\mathcal{A}$ with $a_{i j} \in C(\bar{\Omega})$ and $b_{j}=c=0$ such that the pointed unit disc $\left\{x \in \mathbb{R}^{2}: 0<|x|<1\right\}$ is $\mathcal{A}$-regular even though it is not $\Delta$-regular. The other implication seems to be open (it is known to be false for non-continuous measurable coefficients, see [13]). The fact that the uniform exterior cone property (which is much stronger than $\Delta$ regularity) implies $\mathcal{A}$-regularity (Corollary 3.4 ) had been proved before by Krylov [10, Theorem 5] with the help of partially probabilistic methods. If $\Omega$ is merely $\Delta$-regular, then it seems not to be known whether $\Omega$ is $\mathcal{A}$-regular. Known results concerning this question are based on further restrictive conditions on the coefficients $a_{i j}$. By Theorem 2.1 in connection with Theorem 2.2 we obtain a positive answer for globally Lipschitz continuous $a_{i j}$. The best result seems to be [10, Theorem 4] which goes in both directions: If the $a_{i j}$ are Dini-continuous (in particular, if they are Hölder-continuous), then $\Omega$ is $\Delta$-regular if and only if $\Omega$ is $\mathcal{A}$-regular.

Things are different in the isotropic case (i.e. if $a_{i j}$ is independent of $i, j \in$ $1, \ldots, n)$ : In that case, for merely measurable $a_{i j}$ the operator $A$ is invertible if and only if $\Omega$ is Wiener regular, see [3].

\section{Generation results}

An operator $B$ on a complex Banach space $X$ is said to generate a bounded holomorphic semigroup if $(\lambda-B)$ is invertible for $\operatorname{Re} \lambda>0$ and

$$
\sup _{\operatorname{Re} \lambda>0}\left\|\lambda(\lambda-B)^{-1}\right\|<\infty .
$$

Then there exist $\theta \in(0, \pi / 2)$ and a holomorphic bounded function $T: \Sigma_{\theta} \rightarrow$ $\mathcal{L}(X)$ satisfying $T\left(z_{1}+z_{2}\right)=T\left(z_{1}\right) T\left(z_{2}\right)$ such that

$$
\lim _{n \rightarrow \infty} e^{t B_{n}}=T(t) \text { in } \mathcal{L}(X)
$$

for all $t>0$, where $B_{n}=n B(n-B)^{-1} \in \mathcal{L}(X)$. Here $\Sigma_{\theta}$ is the sector $\Sigma_{\theta}:=$ $\left\{r e^{i \alpha}: r>0,|\alpha|<\theta\right\}$.

If $B$ is an operator on a real Banach space $X$ we say that $B$ generates a bounded holomorphic semigroup if its linear extension $B_{\mathbb{C}}$ to the complexification $X_{\mathbb{C}}$ of $X$ generates a bounded holomorphic semigroup $T_{\mathbb{C}}$ on $X_{\mathbb{C}}$. In that case $T_{\mathbb{C}}(t) X \subseteq X$ (see [12, Corollary 2.1.3]); in particular $T(t):=T_{\mathbb{C}}(t)_{\mid X} \in \mathcal{L}(X)$. We call $T=$ $(T(t))_{t>0}$ the semigroup generated by $B$. It satisfies $\lim _{t \downarrow 0} T(t) x=x$ for all $x \in X$ (i.e., it is a $C_{0}$-semigroup) if and only if $\overline{D(B)}=X$. We refer to [12, Chapter 2] and [2, Sec. 3.7] for these facts and further information.

In this section we consider the parts $A_{c}$ and $A_{0}$ of $\mathcal{A}$ in $C(\bar{\Omega})$ and $C_{0}(\Omega)$ as follows:

$$
\begin{aligned}
D\left(A_{c}\right) & :=\left\{u \in C_{0}(\Omega) \cap W_{\mathrm{loc}}^{2, n}(\Omega): \mathcal{A} u \in C(\bar{\Omega})\right\} \\
A_{c} u & :=\mathcal{A} u \text { and } \\
D\left(A_{0}\right) & :=\left\{u \in C_{0}(\Omega) \cap W_{\mathrm{loc}}^{2, n}(\Omega): \mathcal{A} u \in C_{0}(\Omega)\right\} \\
A_{0} u & :=\mathcal{A} u
\end{aligned}
$$


Thus $A_{c}$ is the part of $A$ in $C(\bar{\Omega})$ and $A_{0}$ the part of $A_{c}$ in $C_{0}(\Omega)$. Note that $D\left(A_{0}\right) \subseteq D\left(A_{c}\right) \subseteq \bigcap_{q>1} W_{\text {loc }}^{2, q}$ by [9, Lemma 9.16]. The main result of this section is the following.

Theorem 4.1. Assume that the $a_{i j}$ are in $C(\bar{\Omega})$ and that $\Omega$ is $\mathcal{A}$-regular. Then $A_{c}$ generates a bounded holomorphic semigroup $T$ on $C(\bar{\Omega})$. The operator $A_{0}$ generates a bounded holomorphic $C_{0}$-semigroup $T_{0}$ on $C_{0}(\Omega)$. Moreover, $T(t) C_{0}(\Omega) \subseteq$ $C_{0}(\Omega)$ and

$$
T_{0}(t)=T(t)_{\left.\right|_{C_{0}(\Omega)}} .
$$

Recall that $\Omega$ is $\mathcal{A}$-regular if one of the following conditions is satisfied:

(a) $\Omega$ satisfies the uniform exterior cone condition or

(b) $\Omega$ is Wiener regular and the coefficients $a_{i j}$ are Dini-continuous.

In particular, $\Omega$ is $\mathcal{A}$-regular if

(a') $\Omega$ is a Lipschitz-domain or

(b') $\Omega$ is Wiener-regular and the $a_{i j}$ are Hölder continuous.

In the following complex maximum principle (Proposition 4.3) we extend $\mathcal{A}$ to the complex space $W_{\text {loc }}^{2, p}(\Omega)$ without changing the notation. We first prove a lemma.

Lemma 4.2. Let $B \subseteq \Omega$ be a ball of center $x_{0}$ and let $u \in W^{2, p}(B), p>n$, be a complex-valued function such that $\mathcal{A} u \in C(B)$. If $\left|u\left(x_{0}\right)\right| \geq|u(x)|$ for all $x \in B$, then

$$
\operatorname{Re}\left[\overline{u\left(x_{0}\right)}(\mathcal{A} u)\left(x_{0}\right)\right] \leq 0 .
$$

Proof. We may assume that $x_{0}=0$. If the claim is wrong, then there exist $\varepsilon>0$ and a ball $B_{\varrho} \subset B$ such that $\operatorname{Re}[\overline{u(x)}(\mathcal{A} u)(x)] \geq \varepsilon$ on $B_{\varrho}$.

Since $\partial_{j}|u|^{2}=\left(\partial_{j} u\right) \bar{u}+u \overline{\partial_{j} u}=2 \operatorname{Re}\left[\partial_{j} u \bar{u}\right]$, and $\partial_{i j}(u \bar{u})=\left(\partial_{i j} u\right) \bar{u}+\partial_{i} u \overline{\partial_{j} u}+$ $\partial_{j} u \overline{\partial_{i} u}+u \overline{\partial_{i j} u}$, and since by ellipticity

$$
\operatorname{Re} \sum_{i, j} a_{i j} \partial_{i} u \overline{\partial_{j} u} \geq 0, \operatorname{Re} \sum_{i, j} a_{i j} \partial_{j} u \overline{\partial_{i} u} \geq 0,
$$

it follows that

$$
\begin{aligned}
\mathcal{A}|u|^{2} \geq & \operatorname{Re} \sum_{i, j} a_{i j}\left(\partial_{i j} u\right) \bar{u}+\operatorname{Re} \sum_{i, j} a_{i j} u \overline{\partial_{i j} u} \\
& +\sum_{j} b_{j} 2 \operatorname{Re}\left[\partial_{j} u \bar{u}\right]+c u \bar{u} \\
\geq & 2 \operatorname{Re}(\mathcal{A} u \bar{u}) \geq 2 \varepsilon \text { on } B_{\varrho} .
\end{aligned}
$$

Let $\psi(x)=|u|^{2}-\tau|x|^{2}, \tau>0$. Choosing $\tau>0$ small enough, we have $\mathcal{A}|\psi| \geq \varepsilon$ on $B_{\varrho}$. 
Since $\psi \in W^{2, p}\left(B_{\varrho}\right) \cap C\left(\overline{B_{\varrho}}\right)$, and since $-\mathcal{A}|\psi| \leq-\varepsilon$ on $B_{\varrho}$, by Aleksandrov's maximum principle [9, Theorem 9.1], see Theorem A.1, it follows that

$$
\begin{aligned}
|u(0)|^{2}=|\psi(0)| & \leq \sup _{\partial B_{\varrho}(0)} \psi \\
& =\sup _{\partial B_{\varrho}(0)}|u|^{2}-\tau \varrho^{2} \\
& \leq|u(0)|^{2}-\tau \varrho^{2}<|u(0)|^{2},
\end{aligned}
$$

a contradiction. For the last inequality we used the hypothesis on $x_{0}=0$.

Proposition 4.3 (complex maximum principle). Let $u \in C(\bar{\Omega}) \cap W_{\mathrm{loc}}^{2, n}(\Omega)$ such that $\lambda u-\mathcal{A} u=0$ where $\operatorname{Re} \lambda>0$. If there exists $x_{0} \in \Omega$ such that $|u(x)| \leq\left|u\left(x_{0}\right)\right|$ for all $x \in \Omega$, then $u \equiv 0$. Consequently,

$$
\max _{\bar{\Omega}}|u(x)|=\max _{\partial \Omega}|u(x)| .
$$

Proof. If $|u(x)| \leq\left|u\left(x_{0}\right)\right|$ for all $x \in \Omega$, then by Lemma 4.2, Re $\left[\overline{u\left(x_{0}\right)}(\mathcal{A} u)\left(x_{0}\right)\right] \leq$ 0 . Since $\lambda u=\mathcal{A} u$, it follows that

$$
\operatorname{Re} \lambda\left|u\left(x_{0}\right)\right|^{2}=\operatorname{Re}\left[\overline{u\left(x_{0}\right)}(\mathcal{A} u)\left(x_{0}\right)\right] \leq 0 .
$$

Hence $u\left(x_{0}\right)=0$.

Next, recall that an operator $B$ on a real Banach space $X$ is called $m$-dissipative if $\lambda-B$ is invertible and

$$
\lambda\left\|(\lambda-B)^{-1}\right\| \leq 1 \text { for all } \lambda>0 .
$$

Now we show that the operator $A_{c}$ is $m$-dissipative and that the resolvent is positive (i.e., maps non-negative functions to non-negative functions).

Proposition 4.4. Assume that $A$ is invertible. Then $A_{c}$ is $m$-dissipative and $(\lambda-$ $\left.A_{c}\right)^{-1} \geq 0$ for $\lambda>0$.

Proof. Let $\lambda>0$. Since by Theorem 3.2 the operator $(\lambda-A)$ is bijective, also $\left(\lambda-A_{c}\right)$ is bijective.

a) We show that $\left(\lambda-A_{c}\right)^{-1} \geq 0$. Let $f \in C(\bar{\Omega}), f \leq 0, u:=\left(\lambda-A_{c}\right)^{-1} f$. Assume that $u^{+} \neq 0$. Since $u \in C_{0}(\Omega)$, there exists $x_{0} \in \Omega$ such that $u\left(x_{0}\right)=$ $\max u>0$. Then by Lemma 4.2, $\mathcal{A} u\left(x_{0}\right) \leq 0$. Since $\lambda u-\mathcal{A} u=f$, it follows that $\Omega$
$\lambda\left(x_{0}\right) \leq f\left(x_{0}\right) \leq 0$ a contradiction.

b) Let $f \in C(\bar{\Omega}), u=\left(\lambda-A_{c}\right)^{-1} f$. We show that $\|\lambda u\|_{C(\bar{\Omega})} \leq\|f\|_{C(\bar{\Omega})}$. Assume first that $f \geq 0, f \neq 0$. Then $u \geq 0$ by a) and $u \neq 0$. Let $x_{0} \in \Omega$ such that $u\left(x_{0}\right)=\|u\|_{C(\bar{\Omega})}$. Then $\left(A_{c} u\right)\left(x_{0}\right) \leq 0$ by Lemma 4.2. Hence $\lambda u\left(x_{0}\right) \leq$ $\lambda u\left(x_{0}\right)-\left(A_{c} u\right)\left(x_{0}\right)=f\left(x_{0}\right) \leq\|f\|_{C(\bar{\Omega})}$.

If $f \in C(\bar{\Omega})$ is arbitrary, then by a) $\left|\left(\lambda-A_{c}\right)^{-1} f\right| \leq\left(\lambda-A_{c}\right)^{-1}|f|$ and so $\left\|\lambda\left(\lambda-A_{c}\right)^{-1} f\right\|_{C(\bar{\Omega})} \leq\|f\|_{C(\bar{\Omega})}$. 
Now we consider the complex extension of $A_{c}$ (still denoted by $A_{c}$ ) to the space of all complex-valued functions on $\bar{\Omega}$ which we still denote by $C(\bar{\Omega})$. Our aim is to prove that for $\operatorname{Re} \lambda>0$ the operator $\left(\lambda-A_{c}\right)^{-1}$ is invertible and

$$
\left\|\left(\lambda-A_{c}\right)^{-1}\right\| \leq \frac{M}{|\lambda|}
$$

where $M$ is a constant. For that, we extend the coefficents $a_{i j}$ to uniformly continuous bounded real-valued functions on $\mathbb{R}^{n}$ satisfying the strict ellipticity condition

$$
\operatorname{Re} \sum_{i, j=1}^{d} a_{i j}(x) \xi_{i} \bar{\xi}_{j} \geq \frac{\Lambda}{2}|\xi|^{2}
$$

$\left(\xi \in \mathbb{R}^{n}, x \in \mathbb{R}^{n}\right.$ ), keeping the same notation, see Lemma 3.3a). We extend $b_{j}, c$ to bounded measurable functions on $\mathbb{R}^{n}$ such that $c \leq 0$ (keeping the same notation). Now we define the operator $B_{\infty}$ on $L^{\infty}\left(\mathbb{R}^{n}\right)$ by

$$
\begin{aligned}
D\left(B_{\infty}\right) & :=\left\{u \in \bigcap_{p>1} W_{\mathrm{loc}}^{2, p}\left(\mathbb{R}^{n}\right): u, \mathcal{B} u \in L^{\infty}\left(\mathbb{R}^{n}\right)\right\} \\
\text { where } \quad B_{\infty} u & :=\mathcal{B} u, \\
\mathcal{B}_{u} & :=\sum_{i, j=1}^{d} a_{i j} \partial_{i j} u+\sum_{j=1}^{d} b_{j} \partial_{j} u+c u \text { for } u \in W_{\mathrm{loc}}^{2, p}\left(\mathbb{R}^{n}\right) .
\end{aligned}
$$

We will show that the operator $B_{\infty}$ is sectorial (Theorem 4.6 below). This is proved in [12, Theorem 3.1.7] under the assumption that the coefficients $b_{j}, c$ are uniformly continuous. We give a perturbation argument to deduce the general case from the case $b_{j}=c=0$. The following lemma shows in particular that the domain of $B_{\infty}$ is independent of $b_{j}$ and $c$.

Lemma 4.5. One has $D\left(B_{\infty}\right) \subset W^{1, \infty}\left(\mathbb{R}^{n}\right)$. Moreover, for each $\varepsilon>0$ there exists $c_{\varepsilon} \geq 0$ such that

$$
\|u\|_{W^{1, \infty}\left(\mathbb{R}^{n}\right)} \leq \varepsilon\left\|B_{\infty} u\right\|_{L^{\infty}\left(\mathbb{R}^{n}\right)}+c_{\varepsilon}\|u\|_{L^{\infty}\left(\mathbb{R}^{n}\right)}
$$

for all $u \in D\left(B_{\infty}\right)$.

Proof. Consider an arbitrary ball $B_{1}$ in $\mathbb{R}^{n}$ of radius 1 and the corresponding ball $B_{2}$ of radius 2. Let $p>n$. Since the injection of $W^{2, p}\left(B_{1}\right)$ into $C^{1}\left(\bar{B}_{1}\right)$ is compact, for each $\varepsilon>0$ there exists $c_{\varepsilon}^{\prime}>0$ such that

$$
\|u\|_{C^{1}\left(\bar{B}_{1}\right)} \leq \varepsilon\|u\|_{W^{2, p}\left(B_{1}\right)}+c_{\varepsilon}^{\prime}\|u\|_{L^{\infty}\left(B_{1}\right)} .
$$

By the Calderon-Zygmund estimate this implies that

$$
\begin{aligned}
\|u\|_{C^{1}\left(\bar{B}_{1}\right)} \leq & \varepsilon c_{1}\left(\left\|B_{\infty} u\right\|_{L^{\infty}\left(B_{2}\right)}+\|u\|_{L^{\infty}\left(B_{2}\right)}\right) \\
& +c_{\varepsilon}^{\prime}\|u\|_{L^{\infty}\left(B_{1}\right)} \\
\leq & \varepsilon c_{1}\left\|B_{\infty} u\right\|_{L^{\infty}\left(\mathbb{R}^{n}\right)}+\left(\varepsilon c_{1}+c_{\varepsilon}^{\prime}\right) \cdot\|u\|_{L^{\infty}\left(\mathbb{R}^{n}\right)} .
\end{aligned}
$$


Since $\|u\|_{W^{1, \infty}\left(\mathbb{R}^{n}\right)}=\sup _{B_{1}}\|u\|_{C^{1}\left(\overline{B_{1}}\right)}$, where the supremum is taken over all balls of radius 1 in $\mathbb{R}^{n}$, the claim follows.

Theorem 4.6. There exist $M \geq 0, \omega \in \mathbb{R}$ such that $\left(\lambda-B_{\infty}\right)$ is invertible and

$$
\left\|\lambda\left(\lambda-B_{\infty}\right)^{-1}\right\| \leq M \quad(\operatorname{Re} \lambda>\omega) .
$$

Proof. Denote by $B_{\infty}^{0}$ the operator with the coefficients $b_{j}, c$ replaced by 0 . Lemma 4.5 implies that $D\left(B_{\infty}^{0}\right)=D\left(B_{\infty}\right)$ and (applied to $\left.B_{\infty}^{0}\right)$ that

$$
\left\|\left(B_{\infty}-B_{\infty}^{0}\right) u\right\|_{L^{\infty}\left(\mathbb{R}^{n}\right)} \leq \varepsilon\left\|B_{\infty}^{0} u\right\|_{L^{\infty}\left(\mathbb{R}^{n}\right)}+c_{\varepsilon}^{\prime}\|u\|_{L^{\infty}\left(\mathbb{R}^{n}\right)}
$$

for all $u \in D\left(B_{\infty}^{0}\right), \varepsilon>0$ and some $c_{\varepsilon}^{\prime} \geq 0$. Since $B_{\infty}^{0}$ is sectorial by [12, Theorem 3.1.7] the claim follows from the usual holomorphic perturbation result [2, Theorem 3.7.23].

Now we use the maximum principle, Lemma 4.2, to carry over the sectorial estimate from $\mathbb{R}^{n}$ to $\Omega$. This is done in a very abstract framework by Lumer-Paquet [11], see [6, Section 2.5] for the Laplacian.

Proof of Theorem 4.1. Let $\omega$ be the constant from Theorem 4.6 and let $\operatorname{Re} \lambda>$ $\omega, f \in C(\bar{\Omega}), u=\left(\lambda-A_{c}\right)^{-1} f$. Then

$$
u \in C_{0}(\Omega) \cap \bigcap_{p>1} W_{\mathrm{loc}}^{2, p}(\Omega) \text { and } \lambda u-\mathcal{A} u=f .
$$

Extend $f$ by 0 to $\mathbb{R}^{n}$ and let $v=\left(\lambda-B_{\infty}\right)^{-1} f$. Then $\lambda v-\mathcal{A} v=f$ on $\Omega$ and $\|\lambda v\|_{L^{\infty}(\Omega)} \leq M\|f\|_{C(\bar{\Omega})}$ by Theorem 4.6. Moreover, $w:=v-u \in C(\bar{\Omega}) \cap$ $\bigcap W_{\mathrm{loc}}^{2, p}(\Omega), \lambda w-\mathcal{A} w=0$ on $\Omega$ and $w(z)=v(z)$ for all $z \in \partial \Omega$. Then by the $p \geq 1$ complex maximum principle Proposition 4.3,

$$
\|w\|_{C(\bar{\Omega})}=\max _{z \in \partial \Omega}|v(z)| \leq \frac{M}{|\lambda|}\|f\|_{C(\bar{\Omega})} .
$$

Consequently,

$$
\begin{aligned}
\|u\|_{C(\bar{\Omega})} & =\|u-v+v\|_{C(\bar{\Omega})} \\
& \leq\|w\|_{C(\bar{\Omega})}+\|v\|_{C(\bar{\Omega})} \\
& \leq \frac{2 M}{|\lambda|}\|f\|_{C(\bar{\Omega})} .
\end{aligned}
$$

This is the desired estimate which shows that $A_{c}$ is sectorial. By [12, Proposition 2.1.11] there exist a sector $\Sigma_{\theta}+\omega:=\left\{\omega+r e^{i \alpha}: r>0,|\alpha|<\theta\right\}$ with $\theta \in\left(\frac{\pi}{2}, \pi\right)$, $\omega \geq 0$, and a constant $M_{1}>0$ such that

$$
\left(\lambda-A_{c}\right)^{-1} \text { exists for } \lambda \in \Sigma_{\theta}+\omega \text { and }\left\|\lambda\left(\lambda-A_{c}\right)^{-1}\right\| \leq M_{1} .
$$


Thus there exists $r>0$ such that $\left(\lambda-A_{c}\right)$ is invertible and $\left\|\lambda\left(\lambda-A_{c}\right)^{-1}\right\| \leq M$ whenever $\operatorname{Re} \lambda>0$ and $|\lambda|>r$. Since $A$ is invertible by Theorem 3.2, it follows that $A_{c}$ is bijective. Since the resolvent set of $A_{c}$ is nonempty, $A_{c}$ is closed. Thus $A_{c}$ is invertible. Since by Proposition $4.4 A_{c}$ is resolvent positive, it follows from [2, Proposition 3.11.2] that there exists $\varepsilon>0$ such that $\left(\lambda-A_{c}\right)$ is invertible whenever $\operatorname{Re} \lambda>-\varepsilon$. As a consequence,

$$
\sup _{\substack{|\lambda| \leq r \\ \operatorname{Re} \lambda>0}}\left\|\lambda\left(\lambda-A_{c}\right)^{-1}\right\|<\infty .
$$

Together with the previous estimates this implies that

$$
\left\|\lambda\left(\lambda-A_{c}\right)^{-1}\right\| \leq M_{2}
$$

whenever $\operatorname{Re} \lambda>0$ for some constant $M_{2}$. Thus $A_{c}$ generates a bounded holomorphic semigroup $T$ on $C(\bar{\Omega})$. Since $D\left(A_{c}\right) \subset C_{0}(\Omega)$ and $\mathcal{D}(\Omega) \subset D\left(A_{c}\right)$ it follows that $\overline{D\left(A_{c}\right)}=C_{0}(\Omega)$. The part of $A_{c}$ in $C_{0}(\Omega)$ is $A_{0}$. So it follows from [12, Remark 2.1.5, Proposition 2.1.4] that $A_{0}$ generates a bounded, holomorphic $C_{0}$-semigroup $T_{0}$ on $C_{0}(\Omega)$ and $T_{0}(t)=T(t)_{\left.\right|_{0}(\Omega)}$ on $C_{0}(\Omega)$.

If the coefficients $a_{i j}$ are merely in $C(\Omega) \cap L^{\infty}(\Omega)$ as in our standing hypothesis we cannot extend the operator to $\mathbb{R}^{n}$ and our proof of analyticity of the semigroup breaks down. But we still obtain a $C_{0}$-semigroup on $C_{0}(\Omega)$

More precisely, we have the following result.

Proposition 4.7. Assume that $\Omega$ satisfies the uniform exterior cone condition. Then $A_{0}$ generates a positive, contractive, irreducible $C_{0}$-semigroup on $C_{0}(\Omega)$. Moreover,

$$
\|T(t)\| \leq M e^{-\varepsilon t} \quad(t \geq 0)
$$

for some $M>0, \varepsilon>0$. The resolvent $\left(\lambda-A_{0}\right)^{-1}$ is compact for all $\lambda \in \varrho\left(A_{0}\right)$.

Proof. By Theorem 2.4 the operator $A$ is invertible. It follows from Proposition 4.3 that $A_{0}$ is $m$-dissipative. Since $D\left(A_{0}\right)$ is dense, by the Lumer-Phillips Theorem the operator $A_{0}$ generates a positive, contractive $C_{0}$-semigroup $\left(T_{0}(t)\right)_{t \geq 0}$ on $C_{0}(\Omega)$. It follows from Theorem A.3 that $D\left(A_{0}\right) \subset C^{\alpha}(\Omega)$. Since the embedding of $C^{\alpha}(\Omega)$ into $C(\bar{\Omega})$ is compact, it follows that the resolvent of $A_{0}$ is compact. Since the resolvent is positive by Proposition 4.3 , it follows from $[2,3.11]$ that the semigroup is positive.

Next show that $u:=\left(\lambda-A_{0}\right)^{-1} f$ is strictly positive if $0 \leq f \in C_{0}(\Omega), f \neq 0$. Assume that $u(x) \leq 0$ for some $x \in \Omega$. Let $v=-u$. Then $\mathcal{A} v-\lambda v=f \geq 0$. It follows from the maximum principle [9, Theorem 9.6] that $v$ is constant. Since $v \in$ $C_{0}(\Omega)$, it follows that $v \equiv 0$. Hence also $f \equiv 0$. This proves the claim. It follows from [16, B-III.3.1] that the semigroup $\left(T_{0}(t)\right)_{t \geq 0}$ is irreducible. [2, Theorem 5.3.8] implies that the semigroup is exponentially stable. 


\section{A. Results on elliptic partial differential equations}

In this section, we collect some results on elliptic partial differential equations, which can be found in text books, for example [9]. We consider the elliptic operator $\mathcal{A}$ from the Introduction.

Theorem A.1 (Aleksandrov's maximum principle, [9, Theorem 9.1]). Let $f \in$ $L^{n}(\Omega), u \in C(\bar{\Omega}) \cap W_{\text {loc }}^{2, n}(\Omega)$ such that

$$
-\mathcal{A} u \leq f .
$$

Then

$$
\sup _{\Omega} u \leq \sup _{\partial \Omega} u^{+}+c_{1}\left\|f^{+}\right\|_{L^{n}(\Omega)}
$$

where the constant $c_{1}$ depends merely on $n$, diam $\Omega$, the ellipticity constant $\Lambda$ and $\left\|b_{j}\right\|_{L^{n}(\Omega)}, j=1 \ldots, n$. Consequently, if $u \in C_{0}(\Omega)$ and $-\mathcal{A} u=f$, then

$$
\|u\|_{L^{\infty}(\Omega)} \leq c_{1}\|f\|_{\left.L^{n}(\Omega)\right)}
$$

and $u \leq 0$ if $f \leq 0$.

For the next two results we assume that the ellipticity constant $\Lambda>0$ is so small that $\left\|a_{i j}\right\|_{L^{\infty}},\left\|b_{j}\right\|_{L^{\infty}},\|c\|_{L^{\infty}} \leq \frac{1}{\Lambda}$.

Theorem A.2 (Interior Calderon-Zygmund estimate, [9, Theorem 9.11]).

Let $B_{2 \varrho}$ be a ball of radius $2 \varrho$ such that $\overline{B_{2 \varrho}} \subset \Omega$, and let $u \in W^{2, p}\left(B_{2 \varrho}\right)$, where $1<p<\infty$. Then

$$
\|u\|_{W^{2, p}\left(B_{\varrho}\right)} \leq c_{\varrho}\left(\|\mathcal{A} u\|_{L^{p}\left(B_{2 \varrho}\right)}+\|u\|_{L^{p}\left(B_{2 \varrho}\right)}\right)
$$

where $B_{\varrho}$ is the ball of radius $\varrho$ concentric with $B_{2 \varrho}$. The constant $\varrho$ merely depends on $\Lambda, n, \varrho, p$ and the continuity moduli of the $a_{i j}$.

Theorem A.3 (Hölder regularity, [9, Corollary 9.29]). Assume that $\Omega$ satisfies the uniform exterior cone condition. Let $u \in C_{0}(\Omega) \cap W_{\text {loc }}^{2, n}(\Omega)$ and $f \in L^{n}(\Omega)$ such that $-\mathcal{A} u=f$. Then $u \in C^{\alpha}(\Omega)$ and

$$
\|u\|_{C^{\alpha}(\Omega)} \leq C\left(\|f\|_{L^{n}(\Omega)}+\|u\|_{L^{2}(\Omega)}\right)
$$

where $\alpha>0$ and $c>0$ depend merely on $\Omega, \Lambda$ and $n$.

In [9, Corollary 9.29] it is supposed that $u \in W^{2, n}(\Omega)$. But an inspection of the proof and of the results preceding [9, Corollary 9.29] shows that $u \in W_{\mathrm{loc}}^{2, n}(\Omega)$ suffices. The above Hölder regularity also holds for solutions of equations in divergence form when the right-hand side $f$ is in $L^{q}(\Omega)$ for some $q>\frac{n}{2}$, see [9, Theorem 8.29]. 


\section{References}

[1] W. ARENDT and PH. BÉNILAN, Wiener regularity and heat semigroups on spaces of continuous functions, In: "Progress in Nonlinear Differential Equations and Their Applications", Vol. 35, Birkhäuser, Basel 1999, 29-49.

[2] W. Arendt, C. BATty, M. Hieber and F. Neubrander, "Vector-valued Laplace Transforms and Cauchy Problems" ( $2^{\text {nd }}$ ed.), Monographs in Mathematics, Birkhäuser, Basel, 2011.

[3] W. Arendt and M. Chovanec, Dirichlet regularity and degenerate diffusion, Trans. Amer. Math. Soc. 362 (2010), 5861-5878.

[4] S. AGMon, A. Douglis and L. NiRENBERG, Estimates near the boundary for solutions of elliptic partial differential equations satisfying general boundary conditions I, Comm. Pure Appl. Math. 12 (1959), 623-727.

[5] S. Agmon, A. Douglis and L. Nirenberg, Estimates near the boundary for solutions of elliptic partial differential equations satisfying general boundary conditions II, Comm. Pure Appl. Math. 17 (1964), 35-92.

[6] W. ARENDT, Semigroups and evolution equations: functional calculus, regularity and kernel estimates, In: "Handbook of Differential Equations Evolutionary Equations", Vol. 1, C. M. Dafermos and E. Feireisl (eds.), Elsevier, 2004, 1-85.

[7] D. Cazenave and A. Haraux, "An Introduction to Semilinear Evolution Equations", Oxford University Press, New York, 1998.

[8] L. C. Evans, "Partial Differential Equations", Amer. Math. Soc., Providence, RI, 1998.

[9] D. Gilbarg and N. S. Trudinger, "Elliptic Partial Differential Equations of Second Order", Springer-Verlag, Berlin, 1998.

[10] N. V. KRYLOV, The first boundary value problem for elliptic equations of second order, Differ. Uravn. 3 (1967), 315-326.

[11] G. Lumer and L. PAQuet, Semi-groupes holomorphes et équations d'évolution, C. R. Math. Acad. Sci. Paris, Sér. A 284 (1977), 237-240.

[12] A. LunARDI, "Analytic Semigroups and Optimal Regularity in Parabolic Problems", Birkhäuser, Basel, 1995.

[13] K. MILLER, Exeptional boundary points for the nondivergence equation which are regular for the Laplace equation and vice-versa, Ann. Scuola Norm. Sup. Pisa 22 (1968), 315-330.

[14] K. MILLER, Nonequivalence of regular boundary points for the Laplace and nondivergence equations, even with continuous coefficients, Ann. Scuola Norm. Sup. Pisa (3) 24 (1970), $159-163$.

[15] G. J. Minty, On the extension of Lipschitz, Lipschitz-Hölder continuous, and monotone functions, Bull. Amer. Math. Soc. 76 (1970), 334-339.

[16] R. NAgEL (ed.), "One-parameter Semigroups of Positive Operators", Lecture Notes in Math., Vol. 84, Springer , Berlin, 1986.

Institute of Applied Analysis

University of Ulm

D - 89069 Ulm, Germany

wolfgang.arendt@uni-ulm.de

Department of Mathematics

Eberhard-Karls-University of Tübingen

D-72076 Tübingen, Germany

schaetz@everest.mathematik.uni-tuebingen.de 